\title{
Double pylorus sign
}

\section{Louise A. Kane • Peter P. Stanich • Veeral M. Oza}

Published online: 15 January 2015

(C) Indian Society of Gastroenterology 2015

The double pylorus (DP) sign is a rare endoscopic finding, with an estimated prevalence of $\sim 0.04 \%$, usually in patients with a history of peptic ulcer disease (PUD)

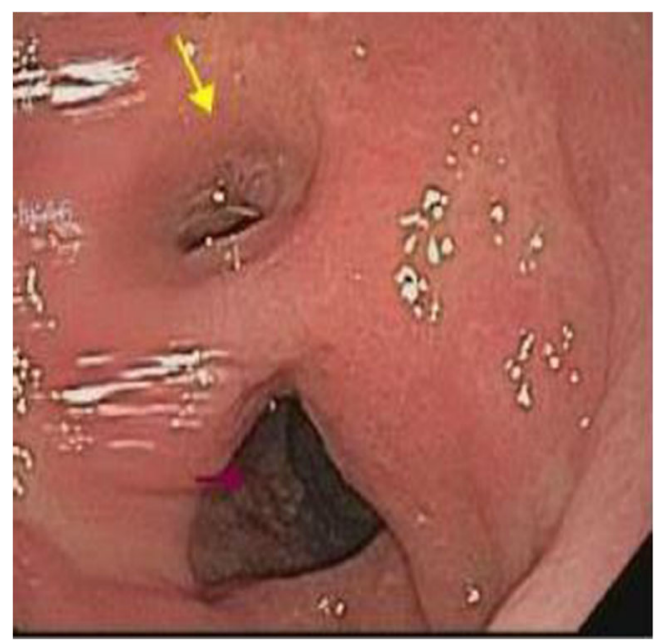

Fig. 1 Double pylorus visualized on endoscopy (arrow shows the second pylorus)

\section{A. Kane}

The Ohio State University College of Medicine,

Columbus, $\mathrm{OH}$, USA

P. P. Stanich • V. M. Oza ( $\square)$

Department of Gastroenterology, Hepatology and Nutrition,

The Ohio State University-Wexner Medical Center,

395 W. 12th Avenue, Columbus, OH 43221, USA

e-mail: veeral.oza@osumc.edu
[1]. This condition is treated with proton pump inhibitors (PPI), and closure of the acquired pylorus has been described [2]. Although this finding can be congenital, a history of increased non-steroidal anti-inflammatory drug (NSAID) use is often elicited, making an acquired etiology the likely diagnosis. The DP finding does not have pathognomonic signs or symptoms, and is often an incidental finding of endoscopy. In some cases, the defect is detectable on radiographic contrast studies as two distinct conduits [1]. Most reported cases of DP arise from gastric ulcers in the antrum of the stomach; rarer yet are reports of this complication from duodenal or gastric cancers [2]. The accompanying image is from a 78-year-old man with a history of PUD, metastatic prostate cancer, and complaints of back pain, fatigue, and melena. Laboratory evaluation revealed anemia with hemoglobin of $6.4 \mathrm{~g} / \mathrm{dL}$. Upper gastrointestinal endoscopy revealed an ulcer in the duodenal bulb and the DP sign within the gastric antrum (Fig. 1). Patient was counseled to avoid all NSAIDs and to use daily PPI indefinitely.

\section{References}

1. Wiseman SM, Tan D, Hill HC. Double pylorus: an unusual endoscopic finding. Endoscopy. 2005;37:277.

2. Akazawa Y, Mizuta Y, Osabe M, et al. A case of double pylorus caused by recurrent gastric ulcers: a long-term endoscopic observation. Dig Dis Sci. 2005;50:2125-8. 